Modern Physics Letters A

(C) World Scientific Publishing Company

\title{
Lowest-order contributions of chiral three-nucleon interactions to pairing properties of nuclear ground states
}

\author{
T. Duguet \\ CEA, Centre de Saclay, IRFU/Service de Physique Nucléaire, F-91191 Gif-sur-Yvette, France \\ National Superconducting Cyclotron Laboratory and Department of Physics and Astronomy, \\ Michigan State University, East Lansing, MI 48824, USA \\ thomas.duguet@cea.fr \\ T. Lesinski \\ Department of Physics and Astronomy, University of Tennessee, Knoxville, TN 37996, USA \\ Physics Division, Oak Ridge National Laboratory, Oak Ridge, TN 37831, USA \\ tlesinsk@utk.edu \\ K. Hebeler \\ TRIUMF, 4004 Wesbrook Mall, Vancouver, BC, V6T 2A3, Canada \\ hebeler@triumf.ca \\ A. Schwenk \\ ExtreMe Matter Institute EMMI, GSI Helmholtzzentrum für Schwerionenforschung GmbH, \\ 64291 Darmstadt, Germany \\ Institut für Kernphysik, Technische Universität Darmstadt, 64289 Darmstadt, Germany \\ TRIUMF, 4004 Wesbrook Mall, Vancouver, BC, V6T 2A3, Canada \\ schwenk@physik.tu-darmstadt.de
}

\begin{abstract}
We perform a systematic study of the odd-even mass staggering generated using a pairing interaction computed at first order in low-momentum interactions. Building on previous work including the (nuclear plus Coulomb) two-nucleon interaction only, we focus here on the first-order contribution from chiral three-nucleon forces. We observe a significant repulsive effect from the three-nucleon interaction. The combined contribution from twoand three-nucleon interactions accounts for approximately $70 \%$ of the experimental pairing gaps. This leaves room for higher-order contributions to the pairing kernel and the normal self-energy that need to be computed consistently.
\end{abstract}

\section{Introduction}

The nuclear energy density functional (EDF) approach is used to study medium-

mass and heavy nuclei in a systematic manner 1. Currently employed parameterizations of the EDF provide a satisfactory description of low-energy properties of known nuclei. However, their empirical character and the spreading of the results obtained from different parameterizations, as one moves away from the valley of stability and enters experimentally unexplored regions, point to the lack of predictive power of today's calculations. 


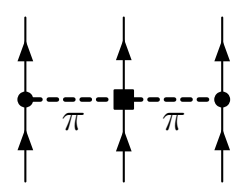

$c_{1}, c_{3}, c_{4}$

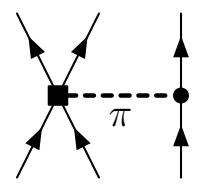

$c_{D}$

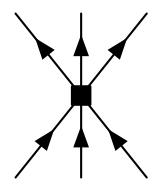

$c_{E}$

Fig. 1. Different contributions to chiral $3 \mathrm{~N}$ forces at $\mathrm{N}^{2} \mathrm{LO}$.

Our objective is to improve on this situation by developing non-empirical EDFs constrained explicitly from inter-nucleon interactions in free space. As a starting point, we performed the first systematic study of ground states of finite nuclei 2134 using a nuclear EDF whose pairing part was obtained from low-momentum twonucleon $(\mathrm{NN})$ interactions $[5] 6$ at first order. The present study builds on these results and focuses on the first-order contributions from chiral three-nucleon $(3 \mathrm{~N})$ interactions. Details are reported elsewhere 7 . Higher-order contributions to the pairing kernel and the normal self-energy are left to future works.

\section{Method}

Our calculations are based on low-momentum interactions, consisting of an NN interaction $V_{\text {low } k}$ with a smooth cutoff $\Lambda=1.8 \mathrm{fm}^{-1}$ and chiral $3 \mathrm{~N}$ interactions $V_{3 \mathrm{~N}}$ at next-to-next-to-leading order $\left(\mathrm{N}^{2} \mathrm{LO}\right)$ with $\Lambda_{3 \mathrm{~N}}=2.0 \mathrm{fm}^{-1}$. The different $3 \mathrm{~N}$ contributions are shown in Fig. 1. For details and for the $3 \mathrm{~N}$ couplings $c_{i}, c_{D}$ and $c_{E}$ used, we refer the reader to Ref. 8 .

Starting from the $\mathrm{N}^{2} \mathrm{LO} 3 \mathrm{~N}$ force, we first construct an antisymmetrized, densitydependent two-body interaction $\bar{V}_{\mathrm{NN}}$ by summing one interacting particle over occupied states in the Fermi sea, extending the calculations of Ref. 9 to general isospin asymmetries. $\bar{V}_{\text {NN }}$ is then added to $V_{\text {low } k}$ to build the first-order pairing interaction from which the anomalous self-energy is computed. To proceed, we generate a high-precision representation of the pairing kernel as a sum of density-dependent separable terms and employ a local density approximation. Details will be given in Ref. 7 .

The remaining part of the nuclear EDF, i.e., the part accounting for the normal self-energy that describes the correlated single-particle motion, is taken as a semiempirical Skyrme parameterization whose isoscalar and isovector effective masses have been constrained from Hartree-Fock calculations of symmetric and pure neutron matter employing the same $\mathrm{NN}$ and $3 \mathrm{~N}$ low-momentum interactions 10 . The present results do not depend significantly on the Skyrme isoscalar effective mass, as long as the value of the latter at saturation density is $0.67-0.73$.

\section{Results and conclusions}

We limit ourselves to discussing the odd-even mass staggering that provides a measure of the lack of binding per particle in an odd isotope/isotone relative to its even 

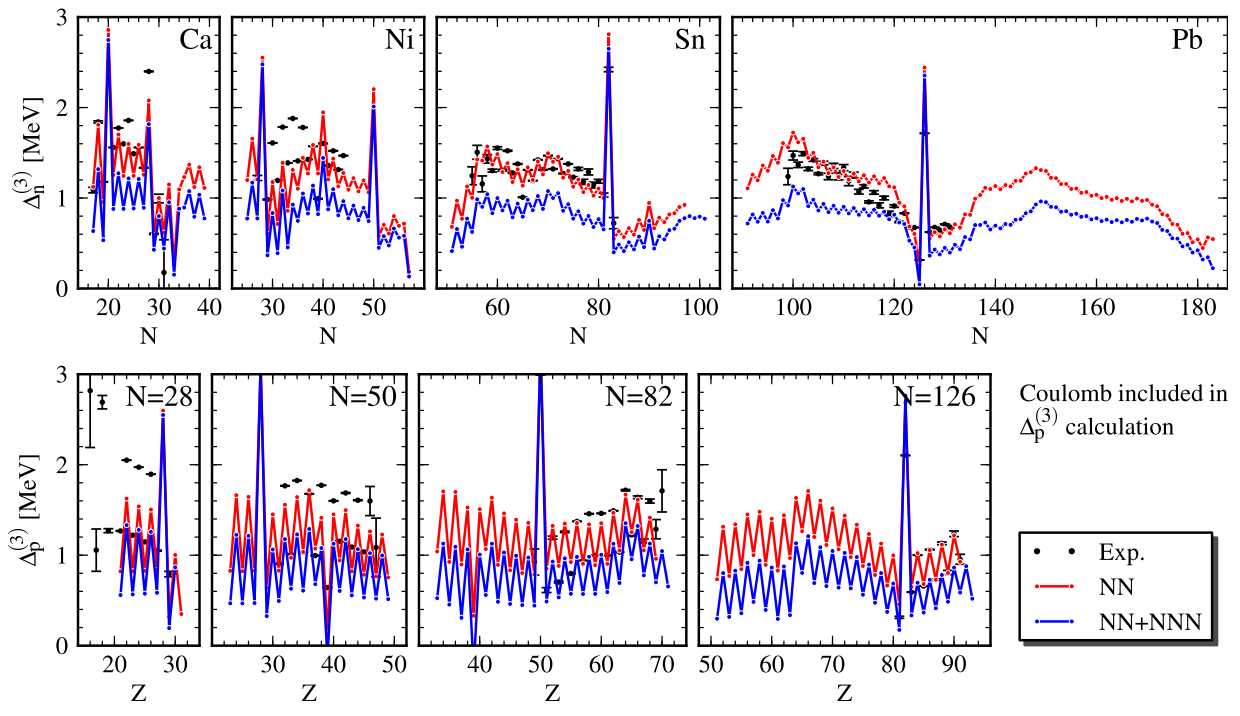

Fig. 2. Experimental and theoretical neutron/proton three-point mass differences along semimagic isotopic/isotonic chains. Results are obtained from a first-order pairing interaction kernel with and without $3 \mathrm{~N}$ contributions.

neighbors; i.e., it essentially extracts the pairing gap 11. Figure 2 displays theoretical and experimental neutron/proton three-point mass differences along several semi-magic isotopic/isotonic chains from proton to neutron drip lines. Results obtained with and without $3 \mathrm{~N}$ contributions to the first-order pairing interaction are compared.

The main result obtained with NN only 4 is that theoretical neutron and proton pairing gaps computed at lowest order are close to experimental ones for a large set of semi-magic spherical nuclei, although experimental gaps are underestimated in the lightest systems. The addition of the first-order $3 \mathrm{~N}$ contribution lowers pairing gaps systematically by about $30 \%$. This is in line with the repulsive character of $\bar{V}_{3 \mathrm{~N}}$ in the ${ }^{1} \mathrm{~S}_{0}$ channel 799 . Although the impact of the $3 \mathrm{~N}$ contribution is rather insensitive to the structure of the particular nucleus under consideration, it displays a slight isovector trend along isotopic (isotonic) chains, most prominently seen in Fig. 2 for the neutron-rich lead isotopes 7 .

The main conclusions of the present work are that (i) it is essential to include $3 \mathrm{~N}$ contributions to the pairing interaction for a quantitative description of nuclear pairing gaps, (ii) the first-order low-momentum results leave about $30 \%$ room for contribution from higher orders, e.g., from the coupling to (collective) density, spin and isospin fluctuations, (iii) in the next steps, the normal self-energy and higherorder contributions to the pairing kernel must be computed consistently starting from the same low-momentum $\mathrm{NN}$ and $3 \mathrm{~N}$ interactions.

This work was supported in part by the U.S. Department of Energy under Contract 
Nos. DE-FG02-96ER40963 and DE-FG02-07ER41529 (University of Tennessee), the Natural Sciences and Engineering Research Council of Canada (NSERC), and by the Helmholtz Alliance Program of the Helmholtz Association, contract HA216/EMMI "Extremes of Density and Temperature: Cosmic Matter in the Laboratory". TRIUMF receives federal funding via a contribution agreement through the National Research Council of Canada.

\section{References}

1. M. Bender, P.-H. Heenen and P.-G. Reinhard, Rev. Mod. Phys. 75 (2003) 121.

2. T. Duguet and T. Lesinski, Eur. Phys. J. Special Topics 156 (2008) 207.

3. T. Lesinski, T. Duguet, K. Bennaceur and J. Meyer, Eur. Phys. J. A40 (2009) 121.

4. T. Duguet and T. Lesinski, AIP Conf. Proc. 1165 (2009) 243, arXiv:0907.1043.

5. S. K. Bogner, T. T. S. Kuo and A. Schwenk, Phys. Rep. 386 (2003) 1.

6. S. K. Bogner, R. J. Furnstahl, S. Ramanan and A. Schwenk, Nucl. Phys. A784 (2007) 79.

7. T. Lesinski, K. Hebeler, T. Duguet and A. Schwenk, in preparation.

8. S. K. Bogner, R. J. Furnstahl, A. Nogga and A. Schwenk, arXiv:0903.3366

9. K. Hebeler and A. Schwenk, arXiv:0911.0483.

10. K. Hebeler, T. Duguet, T. Lesinski and A. Schwenk, Phys. Rev. C80 (2009) 044321.

11. T. Duguet, P. Bonche, P.-H. Heenen and J. Meyer, Phys. Rev. C65 (2001) 014311. 